\title{
Análisis del desarrollo de competencias genéricas: Uso del portafolio de evidencias como
}

\section{estrategia didáctica}

Analysis of the development of generic competences: Use of the evidence portfolio as a teaching strategy

Deida María López Fuentes ${ }^{1 凶}$, Maribel Carmona García ${ }^{2}$, Minerva Reyes Félix² ${ }^{2}$ Diana Elizabeth Valderrábano Pedraza $^{2}$ y Araceli Pineda Moreno ${ }^{3}$

${ }^{1}$ Colegio Preparatorio Semiescolarizado de Xalapa (Xalapa)

${ }^{2}$ Facultad de Estadística e Informática, Universidad Veracruzana (Xalapa)

${ }^{3}$ Facultad de Contaduría Universidad Veracruzana (Tuxpan)

${ }^{凶}$ Autor para correspondencia: deyys@icloud.com

Recibido: $15 / 07 / 2017$

Aceptado: 10/12/2017

\section{RESUMEN}

El presente trabajo pretende contribuir a la identificación y evaluación de las estrategias didácticas que funjan como medio para el desarrollo de las competencias genéricas en alumnos del Nivel Medio Superior, en el área de ciencias sociales. Para evaluar la estrategia didáctica Portafolio de Evidencias, se analizaron diferentes instrumentos de medición existentes con el objeto de aplicarlos al presente trabajo. Sin embargo. Al no encontrar un instrumento ad hoc a los objetivos de esta, se elaboró un cuestionario tipo Likert de 55 ítems que fue aplicado a la muestra seleccionada en dos ocasiones un pretest (antes de utilizar el Portafolio de Evidencias) y en un postest (después de trabajar con el Portafolio de Evidencias). Como resultado de la investigación, se propone al Portafolio de Evidencias como estrategia didáctica que genera el desarrollo de las competencias genéricas, así mismo, el cuestionario elaborado puede ser utilizado para evaluar competencias genéricas con otras estrategias didácticas.

Palabras clave: Competencia, Competencia genérica, Portafolio de evidencias, Estrategia, Prueba de hipótesis.

\begin{abstract}
ABSTRAC
The present work aims to contribute to the identification and evaluation of didactic strategies that serve as a means for the development of generic competences in students of the Higher Level in the area of social sciences. In order to evaluate the Evidence Portfolio didactic strategy, the different measurement instruments were analyzed in order to apply them to the present work. Nevertheless. As it did not find an ad hoc instrument for its objectives, a 55-item Likert questionnaire was developed and applied to the selected sample on two occasions before the test (before using the Evidence Portfolio) and in a postest (after working with the Evidence Portfolio). As a result of the research, the Evidence Portfolio of didactic
\end{abstract}


strategy that generates the development of generic competences is proposed, and the questionnaire elaborated can be used to evaluate generic competences with other didactic strategies.

Keywords: Competition, Generic competition, Evidence portfolio, Strategy, Hypothesis test.

\section{INTRODUCCIÓN}

En las últimas décadas se ha hablado mucho sobre el concepto de competencias. Sin embargo, hasta el momento existe una gran polémica al tratar de definir su enunciación. Uno de los grandes retos a los que se enfrenta este término es el de ser comprendido y valorado. Desafortunadamente se ha generado una polisemia sobre este, ya que no existe realmente una definición única que lo describa. $\mathrm{Al}$ respecto Bisquerra y Pérez (2007) mencionan que esto ha derivado que debido a su complejidad el concepto de competencia esté en revisión.

El término competencia en su sentido más simple y general tiene dos significados, tal como lo describe el diccionario electrónico etimológico; es un sustantivo femenino que pertenece a la familia léxica de dos verbos diferentes, competer y competir.

1. Competer que proviene del latín. competentǐa; cf. Competente, que denota por un lado incumbencia, y por otro poseer la pericia, aptitud, idoneidad para hacer algo o intervenir en un asunto determinado.

2. Competir que proviene del latín competentĭa; cf. Competir, que denota una disputa, oposición rivalidad entre dos a más personas sobre algo, para obtener una misma cosa.

Como se puede apreciar existen dos significados diferentes, pero para lo concerniente al ámbito de educación o pedagogía se utiliza la primera definición, el desarrollar o poseer la pericia, aptitud, idoneidad para hacer algo o intervenir en un asunto determinado.

Acudiendo a la historia, es a finales de los años cincuenta, cuando se comienzan a dar las primeras publicaciones sobre el concepto de competencias. Robert W. White publica en 1959 un artículo en una revista de psicología titulado
"Motivation Reconsidered: The Concep of competence." (La motivación reconsiderada: el concepto de competencia.) Donde define a la competencia como; una habilidad de interactuar de forma efectiva con el contexto o ambiente.

A partir de ese momento las investigaciones sobre las competencias, se comienzan a desarrollar principalmente en el ámbito de la psicología, y el mundo laboral, posteriormente estas se enfocaron sobre la pedagogía, dando como resultado el Aprendizaje Basado en Competencias (ABC), o también llamado Enfoque por Competencias, para fines de esta investigación el término utilizado será el primero.

En lo que respecta a México, este es un concepto relativamente nuevo, ya que es a finales de la década de los noventas cuando se comienzan a dar los primeros acercamientos y esto fue precisamente en la carrera normal. Ya de manera más relevante en el 2002 se comienza a dar la exploración y análisis para la elaboración de un nuevo Programa de Educación Preescolar, y es así como en el 2004 se propone una reforma en la educación preescolar (PEP 2004), donde se establece el primer programa de carácter nacional, organizado a través de competencias. No es, sino hasta en el 2008 que se emite el Acuerdo 442 por el que se establece el Sistema Nacional de Bachillerato en un marco de diversidad, donde se establece como uno de sus ejes el Marco Curricular Común (MCC) con base en competencias. (Vázquez, 2008), lo que deriva en la aplicación del Aprendizaje Basado en Competencias a todos los subsistemas del Nivel Medio Superior.

Por lo anterior, es importante qué primeramente se entienda qué la educación a nivel mundial cambió, giró 180 grados, esto derivado de todos los cambios sociales, económicos, tecnológicos, demográficos y generacionales. Pasó de la 
educación clásica tradicionalista donde lo importante y trascendente era enseñar, a la educación preocupada por generar aprendizaje. Solo entonces se estará en condiciones de poder actuar en pro de ese cambio.

Los estudios o investigaciones que se han dado en relación al $\mathrm{ABC}$ son relativamente pocos. Existe mucha confusión en la forma de aplicarlo dentro y fuera del aula y más aún en la manera de evaluarlo. Se continúa pensando y actuando principalmente en evaluar a los alumnos, tomando como referencias los temas vistos en clase, sin tomar en cuenta las destrezas, habilidades, actitudes y competencias desarrolladas en el proceso de aprendizaje (Hawes, 2005).

Bajo el concepto anterior, nace la necesidad de aplicar una estrategia de aprendizaje organizada desde la perspectiva del ABC. Con el objetivo de generar el desarrollo de las competencias genéricas en los alumnos, así como, promover la calidad y el aprendizaje autónomo, para lo cual se elige la estrategia del Portafolio de Evidencias como medio para generarlo. Con base en lo anterior surge la imperante necesidad de evaluar el desarrollo del Aprendizaje Basado en Competencias, a través de estrategias que puedan ser utilizadas en cualquier asignatura o área. De donde se deriva la siguiente pregunta de investigación; ¿Qué competencias genéricas se generan con el uso de la estrategia didáctica Portafolio de Evidencias?

El Portafolio de Evidencias es un sistema de evaluación que permite evaluar, autoevaluar y valorar, tanto del aprendizaje, como, el desempeño y desarrollo de las competencias del estudiante durante todo el proceso. Tal como lo mencionan Delgado y Oliver (2009) las competencias deben ser desarrolladas y practicadas durante un periodo determinado para poder ser adquiridas y ejercitadas de forma correcta, Por lo que aquel estudiante que mediante un aprendizaje autónomo va poniendo en práctica las competencias siempre contará con una mejor preparación para presentar su evaluación llegado el momento.

\section{MATERIALES Y MÉTODOS}

En este apartado se describen las fases que forman la investigación tales como los instrumentos que se utilizaron para la recolección de datos, la encuesta piloto y sus resultados. Asimismo, se explica el procedimiento realizado para la aplicación de los instrumentos, así como, la depuración de los datos, su categorización y jerarquización correspondiente para proceder a la elaboración del análisis de datos correspondientes. Se elaboró un instrumento (cuestionario tipo Likert) para la presente investigación, tomando como base a los lineamientos establecidos para su construcción por (Núñez et al 2008; Ospina et al. 2005; Hernández et al. 2010). La selección de muestra se realizó con una técnica de muestreo probabilístico, a través del muestreo aleatorio simple, obteniendo como resultado un tamaño de muestra de 67 encuestas.

Con el propósito tanto, de aclarar algunos resultados del cuestionario, como, enriquecer los resultados de la presente investigación se utilizaron los siguientes instrumentos cualitativos: la observación y la entrevista no estructurada. El Cuestionario de Competencias Adquiridas y Desarrolladas, está formado de la siguiente manera; en la primera sección están los datos generales como lo es edad, sexo o grupo, y en la segunda sección 55 ítems tipo Likert. 53 ítems con dirección de lo favorable o lo desfavorable y 2 con dirección de lo desfavorable a lo favorable, con cinco categorías de respuesta; (1) Siempre, (2) Casi siempre, (3) Ocasionalmente, (4) A veces, (5) Nunca.

Se realizó una encuesta piloto para validar el cuestionario, la prueba se aplicó a 10 estudiantes elegidos de manera aleatoria, con los que se actuó, de la misma manera que con la muestra. Solo se les comentó que una vez contestado el cuestionario, se tendría una pequeña plática sobre este con ellos, con el objeto de conocer sus impresiones.

La validación del instrumento de medición se llevó a cabo mediante el coeficiente de Alfa 
Cronbach. La fórmula empleada es la siguiente.

$$
\alpha=\frac{k}{k-1}\left[1-\frac{\sum V_{t}}{V_{t}}\right]
$$

A partir de los cálculos realizados se obtuvo un coeficiente de 0.92; lo que significa que los resultados de opinión de la encuesta piloto de los alumnos respeto a los ítems se encuentran correlacionados de manera altamente confiable y muy aceptable. El coeficiente de Cronbach arrojó que las preguntas planteadas son adecuadas, lo cual junto con la prueba piloto permitió conformar el cuestionario final, el Cuestionario de Competencias Adquiridas y Desarrolladas.

Una vez obtenida la información, se procedió a su captura, para lo cual se diseñó una base de datos compuesta por 67 casos y 55 variables, de los cuales se obtuvo la información a través de este instrumento diseñado para alcanzar los objetivos del presente estudio. La captura de los datos se desarrolló en el software de Microsoft Office Excel 2007, se procede a su chequeo y validación y se utiliza el paquete Statistica, para desarrollar la parte sustancial del análisis estadístico.

\section{RESULTADOS}

Los resultados que se presentan en primera instancia muestran el comportamiento de las competencias de los alumnos. Posteriormente, se indican los resultados referentes a los objetivos planteados de la investigación y finalmente los resultados de la comparación del pretest y postest (mediante la prueba de hipótesis). Después de analizar los resultados obtenidos en ambos test, se puede determinar que se detectó una mejora significativa lo cual se traduce en generación de competencias, aunque en algunas es un poco más marcado el avance, en términos generales se puede considerar que se cuenta con evidencia estadística considerable para determinar la mejora o la generación de algunas competencias. Las competencias que presentan mejora significativa o generación de estas, son las competencias; Instrumentales Lingüísticas, Interpersonales-Individuales y la SistémicasOrganización y Sistémicas-Capacidad emprendedora.

Sin embargo, también se observó una mejora aunque en menor escala en las competencias Instrumentales-Cognitivas e Instrumentales Metodológicas. Las competencias que presentaron un cambio fútil, por lo que no contienen evidencia significativa para considerarlo como mejora o generación de competencias son; las competencias Instrumentales-Tecnológicas, InterpersonalesSociales, Sistémicas-Liderazgo.

Con base a los resultados obtenidos en el cuestionario, en la Tabla 1, se muestra una relación de las variables en las que detectaron una vinculación con el uso del Portafolio de Evidencias, las cuales repercuten en las competencias genéricas para promover la calidad y el aprendizaje autónomo en los alumnos.

Tabla 1. Variables vinculadas con el uso del Portafolio de Evidencias.

\begin{tabular}{|l|l|}
\hline Variables que están vinculadas con el uso del Portafolio de Evidencias y promueven \\
la calidad y el aprendizaje autónomo. \\
\hline \multirow{4}{*}{ Cognitivas } & $\begin{array}{l}\text { Pensamiento; Analítico, Sistémico, Crítico, Reflexivo, } \\
\text { Lógico, Practico, Creativo y Deliberativo. }\end{array}$ \\
\hline \multirow{4}{*}{ Metodológicas } & Gestión de tiempo \\
\cline { 2 - 2 } & Tesolución de problemas \\
\cline { 2 - 2 } & $\begin{array}{l}\text { Orientación al aprendizaje (en el marco pedagógico, } \\
\text { estrategias de aprendizaje) }\end{array}$ \\
\hline
\end{tabular}

Revista Científica Biológico Agropecuaria Tuxpan 5 (2) 


\begin{tabular}{|l|l|}
\hline \multirow{2}{*}{ Lingüísticas } & Comunicación Verbal y \\
\cline { 2 - 2 } & Comunicación escrita \\
\hline \multirow{4}{*}{ Individuales } & Automotivación \\
\cline { 2 - 2 } & Resistencia y adaptación al entorno \\
\cline { 2 - 2 } & Sentido ético \\
\hline \multirow{4}{*}{ Organización } & Gestión por objetivos \\
\cline { 2 - 2 } & Gestión de proyectos \\
\cline { 2 - 2 } & Orientación a la calidad \\
\hline Capacidad & Creatividad \\
\hline Emprendedora & Espíritu emprendedor \\
\cline { 2 - 2 } & Innovación \\
\hline
\end{tabular}

En lo referente a los resultados para la comparación de los resultados del pretest y postest se encontró lo siguiente a través de la hipótesis estadística planteada mediante la prueba del signo para muestras dependientes:

Ho: Los valores de la mediana del pretest de la suma de los ítems son mayores a los valores de la mediana de la suma de los ítems obtenidos del postest (Ho: $\tilde{\mu}$ pretest $>\tilde{\mu}$ postest)

Ha: Los valores de la mediana del pretest de la suma de los ítems son menores a los valores de la mediana de la suma de los ítems obtenidos del postest (Ha: $\tilde{\mu}$ pretest $<\tilde{\mu}$ postest).
Los resultados obtenidos en el paquete Statistica semuestra en la Tabla 2, observando que la prueba del signo no rechaza la hipótesis planteada. El nivel de significancia (error tipo I) que se contempló fue de $\alpha=0.05$.

Según Walpole y Myers (1992) la regla de decisión para verificar si se rechaza o no la hipótesis nula (Ho) es que si $\mathrm{p}<\alpha$, se rechaza Ho. El valor p obtenido fue 0.142639 y el valor de $\alpha=0.05$. Por lo tanto, no se rechaza la hipótesis nula, Ho. Esto es que las sumas totales de los ítems del pretest son mayores al de los ítems del postest. Al ser mayores los valores de los ítems indica que la tendencia de los estudiantes en el pretest es que tuvieron mayor incidencia las respuestas de "a veces" y/o "nunca" y en el postest disminuyó.

Tabla 2. Resultados de la prueba de hipótesis obtenidos de la prueba de signo.

\begin{tabular}{|c|l|l|l|l|}
\hline Par de variables & & $\mathbf{n}$ & $\mathbf{Z}$ & p-value \\
\hline Pretest y Postest & & 67 & 1.466033 & 0.142639 \\
\hline
\end{tabular}

En el contexto de la investigación, significa que hay suficiente evidencia para decir que el uso de la estrategia didáctica Portafolio de Evidencias desarrolla competencias genéricas. Lo anterior coincide con lo establecido en la investigación realizada por Farías y Ramírez (2010) señalada en el marco teórico de la presente investigación, donde concluyen que el Portafolio de Evidencias es una estrategia que diseñada y aplicada debidamente propicia el desarrollo de competencias genéricas.

Por lo tanto se concluye que estadísticamente hay suficiente evidencia para afirmar que el uso del Portafolio de Evidencias genera el desarrollo de competencias, debido a ello, se procederá a determinar cuáles son las competencias más impactadas por este. Para lo cual se utilizó la prueba de Kruskal-Wallis, ya que conforme a lo 
que establecen Walpole y Myers (1992), es la alternativa no paramétrica para una prueba $F$ de análisis de varianza en el caso de comparar tres o más poblaciones.

La aplicación y análisis de la prueba se presenta a continuación:

Ho: No existe diferencia entre las tres competencias.

$\mathrm{Ha}$ : Existe diferencia entre las tres competencias.

El nivel de significancia (error tipo I) usado es de $\alpha=0.05$.
Los resultados obtenidos en el paquete Statistica (ver Tabla 3), muestran que la prueba de Kruskal-Wallis, determina que se rechaza la hipótesis nula (Ho). El valor p que se obtiene es 0.0028 y el valor de $\alpha$ que se propone es de 0.05 . Por lo tanto, se rechaza Ho y se concluye que al menos una de las competencias es diferente. Además se observa que las causantes de esas diferencias son las competencias Interpersonales y Sistémicas ya que son las competencias que el alumno desarrolla más considerando las actividades del Portafolio de Evidencias.

Tabla 3. Resultados de la prueba de hipótesis de la prueba de Kruskal-Wallis.

\begin{tabular}{|l|c|c|}
\hline \multicolumn{2}{|l|}{ Kruskal-Wallis test: $\mathbf{p}=\mathbf{. 0 0 2 8}$} \\
\hline Mediana de las competencias & n (muestra) & Suma de rangos \\
\hline Instrumentales & 67 & 8002.000 \\
\hline Interpersonales & 67 & 6029.000 \\
\hline Sistémicas & 67 & 6270.000 \\
\hline
\end{tabular}

Por la comparación anterior se puede concluir con el análisis inferencial desarrollado que la estrategia didáctica Portafolio de Evidencias, sirve como método de enseñanza detonador para el desarrollo de las competencias genéricas, al mismo tiempo que permite promover la calidad y el aprendizaje autónomo en los estudiantes.

\section{CONCLUSIONES}

Después de analizar los resultados obtenidos con los tres instrumentos de medición utilizados; el cuestionario, la observación y la entrevista no estructurada, se llega a la conclusión que las estrategias didácticas como el Portafolio de Evidencias ayudan de manera significativa al desarrollo de competencias genéricas.

El presente estudio estuvo limitado por el tiempo, por lo que sólo se pudo utilizar la estrategia didáctica Portafolio de Evidencias. Sin embargo, se considera importante mencionar que la muestra siempre estuvo en constante motivación y apoyo. Por lo que en este caso se puede decir que las estrategias pueden ayudar pero no representan el único factor para el desarrollo de las competencias genéricas.

A través de la prueba de signo, se puede concluir que las respuestas en el pretest al arrojar una suma mayor de los ítems indica que la tendencia de los estudiantes antes de trabajar con el Portafolio de Evidencias es que se situaron con mayor incidencia en las respuestas de "a veces" (4) y/o "nunca (5), esto al tener en la escala un mayor valor. Lo cual disminuyó en las respuestas obtenidas en el postest, ya que la suma de estas es menor. Lo cual quiere decir que no se rechaza la hipótesis nula tal como se estableció (Hipótesis nula: Ho: $\tilde{\mu}$ pretest $>\tilde{\mu}$ postest). Lo que estadísticamente se considera como el desarrollo de competencias.

Por otra parte, con el objeto de conocer cuál de las competencias genéricas había tenido o presentado una diferencia con respecto a las otras se procedió a realizar la prueba de KruskalWallis. Donde se concluyó que al menos una de las competencias tiene un comportamiento o desarrollo diferente. Lo anterior se pudo observar en los resultados presentados con base a cada una de las competencias, donde se 
pudo analizar y observar que las competencias menos promovidas fueron las Instrumentales. Y es así como se determinó con la presente prueba que las competencias Interpersonales y Sistémicas son las competencias que el alumno desarrolla más considerando las actividades del Portafolio de Evidencias.

La información vertida en la presente investigación será útil para la institución educativa bajo estudio porque, le ofrece datos y resultados importantes sobre el efecto que estrategias didácticas como el Portafolio de Evidencias genera en sus estudiantes. Por lo que si esta información es utilizada en forma conjunta con la plantilla docente, se pueden generar resultados iguales en otras asignaturas y áreas.

\section{LITERATURA CITADA}

Barberá, E., Gewerc, A., y Rodríguez, J. (2009). Portafolios electrónicos y educación superior en España. Situación y tendencias. Red U-Revista de Docencia Universitaria. Número monográfico III. Portafolios electrónicos y educación superior en España. (En coedición con Red).- /de abril del 2009. Recuperado (22, agosto, 2011) https://doi.org/10.4995/redu.2009.6248

Biggs, J. (2006) Calidad del aprendizaje universitario. ( $2^{\mathrm{a}}$ ed.). Madrid, España: Narcea

Bisquerra, R. (2006). Modelos de orientación e intervención psicopedagógica. ( $\left.5^{\mathrm{a}} \mathrm{ed}.\right)$. Madrid: España. Praxis.

Bisquerra, R. y Pérez, N. (2007). Stel.ub.edu. Recuperado (20, agosto, 2011) de http://stel.ub.edu/grop/files/Competencias emocionales-P.pdf

Briones, G. (2006). Teorías de las ciencias sociales y de la educación: Epistemología. México: Trillas

Cantisani, M. (2006). Factores atribuibles al estudiante de preparatoria que contribuyen al logro de su éxito académico. Tesis de maestría, Instituto Tecnológico y de Estudios Superiores de Monterrey. Recuperado el 26 de septiembre de 2011, de http://biblioteca.itesm.mx/cgibin/doctec/ope $\underline{\text { ndoc} ? \text { cual }=5448 \& \text { archivo }=120702 \& \text { pagina }}$ $=14741 \&$ paginas $=14741,2,3 \&$ query=autor $\% 3 \mathrm{D}$ (cantisani

Díaz Barriga, A. (2006). El enfoque de competencias en la educación: ¿Una alternativa o un disfraz de cambio? Revista Perfiles Educativos, 28(111), 7-36.

Díaz Barriga, F. y Hernández, G. (2002). Estrategias docentes para un aprendizaje significativo. Una interpretación constructivista. (2a ed.). Distrito Federal. México: McGraw Hill.

Estévez, H. E. (2002) Enseñar a aprender. Estrategias Cognitivas. Distrito Federal, México. Paidós.

Frade, L. (2009). Desarrollo de competencias en educación: desde preescolar hasta el bachillerato. ( $2^{\mathrm{a}}$ ed.). Distrito Federal, México: Inteligencia educativa.

Farías, G. y Ramírez. M. (2010). Desarrollo de cualidades reflexivas de profesores en formación inicial a través de Portafolios Electrónicos. Revista Mexicana de Investigación Educativa, 15(44),141-162.

Gallego, D., Cacheiro, Martín, A. y Angel, W. (2009). El Portafolio como estrategia de enseñanza y aprendizaje, EDUTEC. Revista Electrónica de Tecnología Educativa (30). https://doi.org/10.21556/edutec.2009.30.444

García, B. y Baena, R. (2009). El uso de portafolios en la docencia universitaria: experiencia de renovación metodológica en la asignatura de Geomorfología fluvial. (Titulación de geografía). Revista Iberoamericana de Educación, 49(3), 1-11. https://doi.org/10.35362/rie4922108

García, J., Terrón M. y Blanco, Y. (2010) Desarrollo de recursos docentes para la evaluación de competencias genéricas. Revisión, Revista AENUI, 3(2).

Guil, M. (2006). Escala mixta Likert-Thurstone. Revista Andaluza de ciencias sociales (5). Consultado del (20, agosto, 2011) de http./dialnet.unirioja.es/servlet/fichero_artic ulo? codigo $=2151095$

Hernández, P., García M., Martínez, P., Hervas, R. y Maquillón, J. (2002). La constancia entre motivos y estrategias de aprendizaje en estudiantes universitarios. Revista de investigación educativa, 20(2), 487-510. 
Hernández, R., Fernández, C., y Baptista, L. (2010). Metodología de la investigación. (5 $5^{\mathrm{a}}$ ed.). Distrito Federal, México: McGraw Hill.

Martínez, M. (2009). El portafolio para el aprendizaje y la evaluación. Madrid, España: Edit.um

Mellado, M. (2010). Portafolio en línea en la formación docente. Revista Electrónica de Tecnología Educativa (12). Consultado del (20, agosto, 2011) de http://redalyc.uaemex.mx/src/inicio/ArtPdf Red.jsp?iCve $=15513269007$

Mendenhall, W., Wackerly, D. y Scheaffer, R. (1994). Estadística Matemática con Aplicaciones. (2 ${ }^{\text {a }}$ ed.). Distrito Federal, México: Grupo Editorial Iberoamericana.

Moreno, T. (2010). El currículo por competencias en la universidad: Más ruido que nueces. Revista de la Educación Superior, 39(154), 77-90.

Murillo, G. (2009). Evaluación del desempeño docente desde competencias genéricas en la universidad de Costa Rica. Revista Electrónica, 9 (1), 1-25.

Navarro, Y., Pereira, M., Pereira, L., Fonseca, N., y Fonseca, N. (2010). Una mirada a la planificación estratégica curricular. Telos Revista de Estudios Interdisciplinarios en Ciencias Sociales, 12(2), 202-216.

Núñez, R., Rodríguez, J. y Solares, A, (2008). Elaboración de un cuestionario para la evaluación de competencias genéricas en estudiantes universitarios. Apuntes de Psicología, 26 (1), 35-49.

Ortiz, J., Rendón, M. y Atehortúa, J. (2009). Sobre la necesidad de un nuevo enfoque para la identificación, medición y desarrollo de competencias. Revista

Ciencias Estratégicas, 17, 243-250.

Ospina, B., Sandoval, J., Aristizábal, C. y Ramírez, M. (2005). La escala de Likert en la valoración de los conocimientos y las actitudes de los profesionales de enfermería en el cuidado de la salud. Antoquia. Revista de Investigación y Educación en enfermería, 23(1), 14-29.

Pereda, S. y Berrocal, F. (1999). Gestión de recursos humanos por competencias. Madrid. España: Centro de estudios Ramón y Areces.

Perrenoud, Ph. (2008, Junio). Construir las competencias, ¿es darle la espalda a los saberes? Red U. Revista Docencia Universitaria, (monográfico II). Recuperado el agosto, 06, 2011 https://doi.org/10.4995/redu.2008.6270

Ramírez, C. y Vales, J. (2005). Competencias docentes asociadas al desempeño académico en matemáticas en estudiantes del nivel medio superior. Revista Vasconcelos de Educación, 1 (1) 11-19.

Rodríguez, G., Gil, J. y García, E. (1996).Metodología de la investigación cualitativa. Málaga: Ediciones ALJIBE.

Serena, M. (2011). Colegio Preparatorio Semiescolarizado de Xalapa. Consultado el (20, octubre, 2011) de http://www.preparatoriajuarez.edu.mx

Scheaffer, R. Mendenhall, W. y Ott, L. (1987). Elementos de muestreo. Distrito Federal, México: Grupo editorial Iberoamérica.

Tobón, S. (2006). Uv.mx. Recuperado (08, febrero, 2011) de www.uv.mx/facpsi/proyectoaula/documents $\underline{\text { Lectura5.pdf }}$

Vázquez, J. (2008). Acuerdo número 442 por el que se establece el Sistema Nacional de Bachillerato en un marco de diversidad. Diario Oficial de la Federación del 26 de septiembre del 2008. Consulta realizada el 09 de febrero del 2011 en

http://www.reformaiems.sems.gob.mx/wb/riems/acuerdos secr etariales

Villa, A. y Poblete, M. (2010). Aprendizaje basado en competencias: Una propuesta para la evaluación de las competencias genéricas. (3aed.). Bilbao, España: Universidad de Deusto.

Villardón, L. (2006). Evaluación del aprendizaje para promover el desarrollo de las competencias. Educatio Siglo XXI, 24 57-76. Recuperado de

http://revistas.um.es/educatio/article/view/1 53/136

Walpole, R. y Myers, R. (1992). Probabilidad y estadística Modelos. (2a ed.). Distrito Federal. México: McGraw Hill. 
López et al., 2017

White, R. (1959). Motivation reconsidered: The concept of competence. Psychological Review, 66(5), 297-333.

https://doi.org/10.1037/h0040934
Wielenga, D. (2002). Probing and Proving competence. Recuperado de http://repub.eur.nl/res/pub/1224/2\%20Probi ng\%20and\%20Proving\%20Competence.pdf

Copyright (c) 2017 Deida Maria López Fuentes, Maribel Carm ona Garcia, Minerva Reyes Félix,

Diana Elizabeth V alderrábano Pedraza y Araceli Pineda Moreno

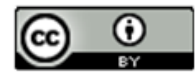

Este tex to está protegido por una licencia licencia Creative Commons 4.0

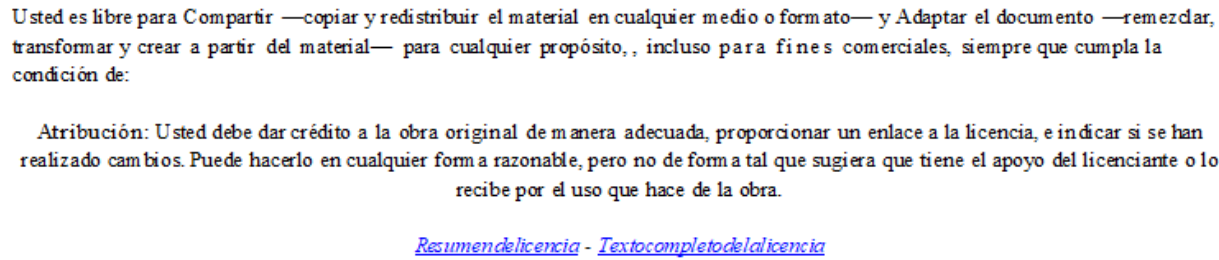

Resumendelicencia - Textocompletodelalicencia 